\title{
Monolithic piezoceramic actuators with a twist
}

\author{
Jikun Yang ${ }^{1,2,3^{*}}$, Faqiang Zhang ${ }^{2}$, Yongxiang $\mathrm{Li}^{2}$, Zhifu Liu $^{2^{*}}$ and Shuxiang Dong ${ }^{1^{*}}$
}

\begin{abstract}
Designing artificial structures with heterogeneous elements and manipulating their interface coupling ways usually bring in synthetic neo-nature to functional devices. For piezoceramic devices, the deformation response refers to a variety of extensional, contractional, or shear modes of crystals, and also relies on boundary conditions from morphology design. However, to pursue fundamental torsion actuation in an integrated piezoceramic component is still a long-term tough task due to nil twist mode limited by microscopic crystal mirror symmetry. Herein, we demonstrate a design of cofired monolithic actuators to originally overcome this obstacle. The prototype device is composed of two sets of stacked actuation subunits that work on artificially reverse face shear modes, and their chiral stiffness couplings will synergistically contribute to synthetic twist outputs at a broad bandwidth. Finite element simulation reveals twist displacements are highly tunable by manipulating the geometrical dimensions. Transverse deflection measurements manifest the stable and sizeable linear actuation response to applied electric fields (around $3.7 \mu \mathrm{m}$ under $40 \mathrm{~V}$ at $1 \mathrm{~Hz}$ ). Importantly, the design actually introduces a more general route to enable arbitrary modes and actuation states in integrated piezoceramic components.
\end{abstract}

Keywords: integrated piezoelectric devices, twist mode, cofired manufacture, precision actuation, ordered structure

\section{INTRODUCTION}

Piezoelectric actuators and micromotors enable movements and positioning up to the nanometer resolution in advanced microelectromechanical systems such as laser radar scanners and photolithographic focusing units [1-4]. By means of piezoelectric crystal elements, highprecision drive applications have come into reach that requires rapid reactions, high specific energy density, and immunity to electromagnetic interference [5-7]. For practical actuation, piezoelectric device design and electric or mechanical boundary conditions are heavily related to the operating mode of elastomer components, where key piezoceramic units essentially provide the fundamental stretch or shear drive strain [8-10]. Complex elastic deformation states enabled by piezoceramics are achieved by adjusting the drive frequencies to excite the corresponding high-order resonant response (restricted by a narrow working bandwidth) [3,11], or through elastic couplings by virtue of distributed actuation patches bonded to mechanical components (with service fatigue problems) [12-14]. Piezoelectric twist elements, working on a most basic deformation mode, are widely used to provide precision torsion actuation, and to actively perceive or compensate uncontrolled rotating vibration and noise in smart mechanical structures such as aircraft blades, and flexible surgical robot arms. However, the existing manufacturing processes are extremely complicated and fail to meet the requirements of miniaturization and industrialized mass production [14-17].

In essence, rotation movements of piezoelectric devices are excited by creating torque moments induced from the asymmetrical structure design, which substantially means converting normal stretch and shear deformation modes of piezoceramic actuation units into the equivalent twist type $[9,13,18-20]$. One specific method is to imitate the motion principle of chiral helical structures. Mohammadi et al. [21] proposed a spiral-shaped piezoceramic actuator, which could accumulate and amplify the tangential stretch strain of $\mathrm{d}_{31}$ mode and output effective rotational displacements. Later studies improved the heteroideus morphology by designing cylinder piezoceramic elements and distributed spiral electrodes to guarantee mechanical rigidity [22]. Another typical route directly builds a torsional strain based on naturally

\footnotetext{
${ }^{1}$ Department of Materials Science and Engineering, College of Engineering, Peking University, Beijing 100871, China

${ }^{2}$ CAS Key Laboratory of Inorganic Functional Materials and Devices, Shanghai Institute of Ceramics, Chinese Academy of Sciences, Shanghai 200050, China

${ }^{3}$ School of Electrical Engineering and Automation, Hefei University of Technology, Hefei 230009, China

* Corresponding authors (emails: jkyang@pku.edu.cn (Yang J); liuzf@mail.sic.ac.cn (Liu Z); sxdong@pku.edu.cn (Dong S))
} 
occurring $\mathrm{d}_{15}$ shear mode. Glazounov et al. [23] designed a tube actuator consisting of even strip piezoceramic segments polarized along the axial direction and jointed by adhesive electrodes in accord with the circumference, and their shear deformation would synergistically behave as an effective torsion strain in the end face. A more compact mono-morph twist plate actuator composed of two sets of glued $d_{15}$ shear patches polarized oppositely, is proposed, where torque moments arise from their reverse-direction shear deformation [24]. Besides piezoceramics, Berik et al. [25] obtained direct torsion actuators using bimorph-like piezoelectric single crystals working on $d_{36}$ face shear modes. However, these complex devices all rely on stiffness couplings based on fragile structural adhesive (with strain reaction problems and much lower Young's modulus), which, as the main technique obstacle for twist actuators [10,24,26,27], determines that their service performance will heavily suffer from displacement hysteresis, poor usable life, and unavailable miniaturized integratability.

Monolithic piezoceramic components based on lowtemperature multilayer cofired ceramic technology (LTCC) are one of the mainstream production ways of piezoelectric devices, especially, with complex morphologies in an integrated plate $[8,28-31]$. However, restricted by perpendicular applied electric fields and polarization of $d_{15}$ shear mode, neither piezoceramic twist plate actuators would support LTCC processes. Until recently, systematic methodologies on piezoelectric metamaterials and devices with ordered substructures have been proposed, which reveals that apparently macroscopic symmetry and synthetic deformation states of piezoceramics may be artificially rebuilt with the aid of patterned electrodes and arrayed piezo-units [32,33], and basic sheartype cofirable piezoceramic components are successfully invented [8]. These advances theoretically set the tone for further realizing twist actuation without the aforementioned puzzling weaknesses.

In this study, we have developed a fresh design to fulfill the fundamental twist mode in an integrated actuation component. The monolithic actuator is composed of cofired bimorph-like dual multilayer face-shear elements, where arrayed piezoelectric subunits are orderly stacked and enabled by patterned electrodes. Dual elements work on inverse face shear states, and thus their stiffness couplings lead to synthetic twist motion. A stable and sizable effective deflection output is detected $(3.7 \mu \mathrm{m}$ under $40 \mathrm{~V}$ at $1 \mathrm{~Hz}$ ). This design paves the way for cofired integration and mass production of piezoelectric twist actuators based on mature LTCC technology, and its methodology also reveals that by constructing identical function subunits at the bottom and rebuilding apparent symmetry, complex extrinsic piezoelectric modes are entirely expected to come into reality in one monolithic component.

\section{EXPERIMENTAL SECTION}

\section{Preparation of cofired monolithic twist actuators}

Commercial raw PZT-5H piezoceramic powder (Xi'an Kanghong cooperation, with large signal $d_{33}$ around $600 \mathrm{pm} \mathrm{V}^{-1}$ ), polyvinyl alcohol (binder), di- $n$-octyl orthophthalate (plasticizer), and ethyl acetate (solvent) were ball-mixed together for $24 \mathrm{~h}$ to get homogeneous precursor slurry. The raw slurry was tape cast to form embryo thick plasticized films. After blank films were cut into smaller standard sheets and punched with location holes, patterned electrodes were precisely screen printed on them. Sequenced stacked sheets were laminated and compressed into one unibody by hot-isostatic pressing under the pressure of $40 \mathrm{MPa}$ at $70^{\circ} \mathrm{C}$ for $30 \mathrm{~min}$. Diced individual intermediate components were slowly burned up to $600^{\circ} \mathrm{C}$ to remove organic ingredients, and sintered for crystallization under $950^{\circ} \mathrm{C}$ for $3 \mathrm{~h}$ to get final cofired multilayer piezoceramic specimens. The side surfaces of specimens were polished to expose inner electrodes, and external silver electrodes were prepared with the postfire process at $650^{\circ} \mathrm{C}$ for $0.5 \mathrm{~h}$. Notably, the external ports should be elaborately printed to avoid any electrically short circuit induced by the narrow spacing in between. Wires were welded to meet driving demands, and specimens were finally polarized in silicone under a directcurrent electric field of $30 \mathrm{kV} \mathrm{mm}^{-1}$ for $20 \mathrm{~min}$ as design.

\section{Microscopic structure and actuation performance}

A twist actuator and its corresponding torsion deformation state highly rely on accurate multilayer morphologies. The inner microscopic structures of patterned electrodes and piezoceramic layers were observed by scanning electron microscopy (SEM, Zeiss Merlin Compact), and element distributions were detected by energy dispersive spectroscopy (EDS). The frequency dependence of impedance was tested by an impedance analyzer (4294A, Agilent Technologies). Since only one channel drive signal was provided, the experimental curve was detected under a single drive way. To quantitatively evaluate twist outputs of the monolithic actuator, one side surface was attached to a three-dimensional-printed optical platform, and the real-time transverse deflection of another side was measured by a laser feedback interferometer (LeiCe LY1000). During measurements, dual- 
channel drive signals were provided by a signal generator (Tektronix AFG3022B), amplified by a low-frequency amplifier (PINTEK HA-405), and monitored by an oscilloscope (Keysight MSOX4024A). The displacement outputs were automatically recorded with the aid of LabVIEW codes.

\section{Numerical finite-element calculation}

The twist deformation and the corresponding displacement outputs or von Mises stress of monolithic actuators at serial frequencies and drive voltages were calculated by a finite-element method (COMSOL codes). Numerical models were simplified by reasonably disregarding the edge regions between actuation strain subunits. During pretreatments, $\mathrm{PZT}-5 \mathrm{H}$ piezoceramic was selected for the corresponding model domain and physical fields, and polarization of each subunit was separately set as design. Driving frequencies, voltages, and geometrical sizes were processed as parameterized values. One side surface was mechanically fixed, and actuation vertexes in another side were adopted as motion points to calculate the displacement outputs and effective rate of twist. The torsion deformation and impedance response were investigated by the frequency response module.

\section{RESULTS AND DISCUSSION}

The design principle starts from the ideology that by orderly organizing piezoelectric actuation subunits in suitable spatial parts of an integrated component, ex- trinsic synthetic deformation states of piezoceramics could be created [32,33]. Theoretically, to excite twist modes in an elastic body, an effective torque moment relative to its fixed end is necessary. The specific method in this original design is illustrated in Fig. 1a. A bimorphlike piezoelectric structure is divided into two layers that are each composed of four actuation subunits. Quadrunits in each layer diagonally work on transverse extensional or contractional states, so their distortions give rise to one elongated diagonal and another shrunken one, and further induce synergetic face shear deformation (Fig. S1). Notably, the two layers are driven by inversephase signals and their shear deformations occur in exactly opposite directions, which means the crystal mirror symmetry about the neutral surface of piezoelectric elastomers is actually apparently broken. From the view of the side face, a torque moment is provided, and the whole monolithic actuator will exhibit a synthetic twist response.

The artificial face shear mode in piezoceramics means much more than an alternative route to excite the twist response, and in fact, the reasonable utilization of that is the key precondition to achieve monolithic fabrication of the twist actuator due to its compatibility with LTCC technology, where polarization and applied electric fields should be all along with the device thickness directions. For schematic design here, each piezoceramic layer in Fig. 1a is adjusted as three layers and one strain buffer layer is set up in the middle (Fig. 1b). Notably, besides
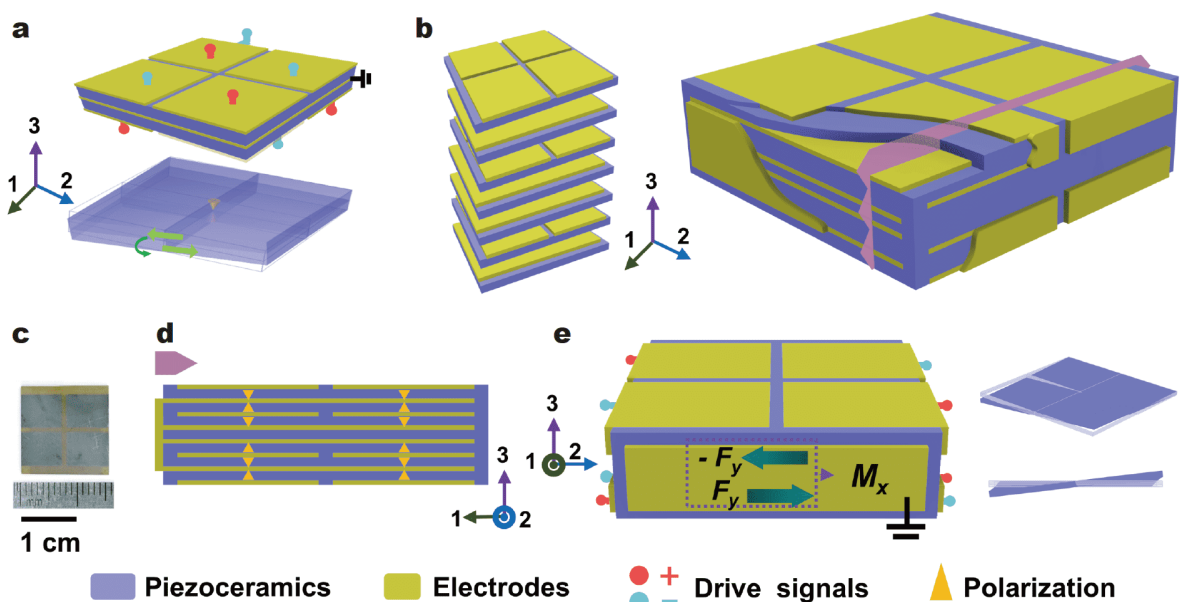

e

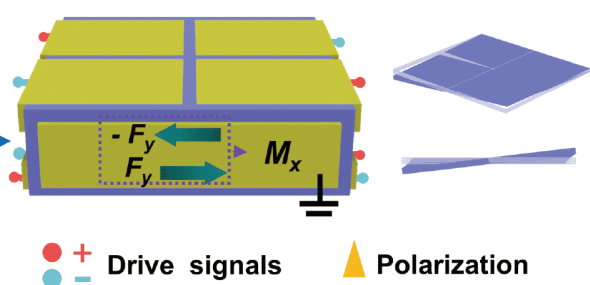

Figure 1 Schematic design and working principle of the cofired monolithic twist actuator. (a) The specific method to excite torsion deformation in one integrated component. Dual piezoceramic layers each composed of four subunits operate on reversed artificial face shear mode, and their distortions lead to an effective moment of torque force and twist deformation. (b) The structure design of a multilayer monolithic twist actuator. It is achieved by tailoring two types of patterned electrodes and arranging their piezoceramic subunits in order with the aid of LTCC technology. (c, d) The appearance image and inner interdigital polarization of the actuator. (e) Under unique reverse-phase dual-drive signals, the monolithic actuator will excite twist deformation. 
conventional full electrodes as ground ends, around half inner electrodes are tailored and screen-printed as patterned morphologies composed of four discrete subparts. The preparation processes are standard LTCC procedures and detailly expounded in the EXPERIMENTAL SECTION (Fig. S2). After ordered lamination and cofired treatment of raw embryo, inner electrodes are connected out in electrically parallel with the aid of nine pieces of postfire terminal ports on side surfaces (Fig. 1b). The prototype monolithic specimen is displayed in Fig. 1c and its geometrical dimensions are illustrated in Fig. S3. Fig. 1d presents the inner interdigital polarization states, which, together with applied electric fields, are both along with compulsory thickness directions. Six active layers output effective strain, and a middle buffer layer behaves as a strain gradual-transition area to avoid micro-cracks, which is especially necessary in situations of tiny thickness. The specific drive method is displayed in Fig. 1e. Besides common ground, the other eight external ports are divided into two groups and driven by dual inversephase signals. When the top three layers and bottom three layers respectively work on face shear modes in opposite directions, finite element analysis (FEA) confirms its synthetic twist deformation.

As a thin-walled plate elastomer, effective twist outputs of the monolithic actuator should be highly related to its torsional rigidity, which is further determined by geometrical dimensions. To investigate the actuation performance, FEA and theoretical analysis were combined to quantificationally evaluate the transverse twist deflection magnitudes and the corresponding influence factors (see Fig. 2). With the structure, length, and width of the ac- tuator fixed, the twist displacements versus the applied voltage and layer thickness are preliminarily calculated in Fig. 2a. The outputs linearly increase with drive voltages, while they present obvious nonlinear variation tendency along with layer thickness. When the thickness reduces, the twist displacements sharply rise to sizable values around dozens of micrometers. Here, we define the effective rate of twist $\theta_{\text {eff }}$ as

$\theta_{\text {eff }}=\frac{2 u}{l w V}$,

where $l$ and $w$ are the length and width of monolithic actuators, and $u$ is the maximum values of transverse deflection. The definition formula has a denominator item of applied voltages $V$ to focus on essential dimension influence factor under unit drive signals. Fig. $2 \mathrm{~b}$ visually plots the calculated $\theta_{\text {eff }}$ values along with increasing thickness-to-length ratio (TLR) of actuators, and these points indicate an obvious nonlinear relationship. From the perspective of continuum mechanics, the actuator is a narrow rectangular section bar with torsion deformation, and based on membrane analogy, its rate of twist could be theoretically predicted as

$\theta_{\text {eff }}=\alpha t^{-3}$,

where $\alpha$ and $t$ are the comprehensive scale factor and thickness of each layer, respectively. Considering the length and width of simulation models are constant, $\theta_{\text {eff }}$ is supposed to be inversely proportional to the third power of the TLR. When fitted as the theoretical prediction, a well-matched curve to simulated results appears in Fig. 2b. This indicates that the actuator is a highly tunable design, where the response is supposed to be effectively
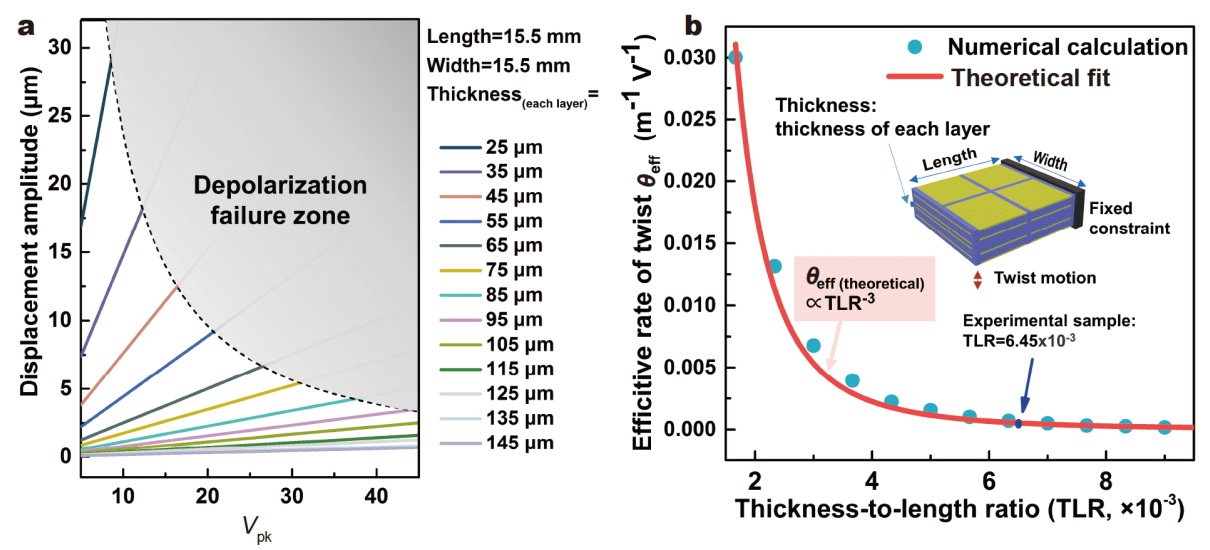

Figure 2 Displacement output characteristics and its dependence of geometrical dimensions of the monolithic twist actuators. (a) Displacement amplitudes linearly increase with drive voltages, and have a heavily nonlinear relationship with layer thickness. Tunable and quite sizable outputs could be obtained by manipulating geometrical dimensions. (b) The numerical calculated effective rate of twist is inversely proportional to the third power of the TLR (the thickness means thickness of each layer), which agrees well with theoretical results of torsional narrow rectangular section bars and indicates good design controllability. 
manipulated by adjusting the geometrical sizes. For the prototype specimen here, its length, width, and the thickness of each layer are $15.5 \mathrm{~mm}, 15.5 \mathrm{~mm}$, and $100 \mu \mathrm{m}$, respectively, so the TLR value is about $6.45 \times$ $10^{-3}$.

The microscopic multilayer morphologies of a twist actuator, especially its unique structure of sequenced patterned electrodes inside guarantee the final deformation outputs. As a demonstration, the microstructures of prototype specimens are checked by SEM and EDS methods. The thickness of piezoceramic layers is observed uniform and as around $100 \mu \mathrm{m}$ each (Fig. 3a), and two types of electrodes are well alternately arranged and aligned in longitudinal rows as design. Cross-section images also indicate the piezoceramic layers form a compact whole without observable cracks, and the spaceconfined electrodes are flat embedded between them (Fig. 3b). The amplified microregion presents piezoceramics are fine crystalized with mean grain sizes around $2.3 \mu \mathrm{m}$ (Fig. 3c). The electrodes are about $3 \mu \mathrm{m}$ thick, which show clearly and tightly bound interfaces between adjacent piezo-layers. These fine constituents and inner morphologies physically ensure objective synthetic outputs and fine piezoelectric response.

For classical piezoelectric continuum elastomers, twist vibration could be excited only at a very narrow frequency range, which is exactly the resonant torsion state $[3,8,34,35]$. As a comparison, the novel design here relies on the synergetic deformation of arrayed piezoceramic subunits instead of mechanical resonance, so the prototype monolithic actuator is able to provide twist actuation from a static state to wider broadbands. Its stable twist response and stress distribution dependence upon driving frequencies are demonstrated by finite-element simulation (Fig. 4a and Fig. S4). When the drive frequency gets close to the resonant one, twist deformation turns to be more ideal and stress distributes more uniformly with milder gradients due to the equipartition of elastic energy. Impedance spectroscopy around resonant states was further experimentally measured. A peak value appears at $2764 \mathrm{~Hz}$, which matches well with the calculated $2585 \mathrm{~Hz}$ (Fig. 4b). These numerical simulations and calculations have shown that the design successfully excites twist outputs in a monolithic piezoelectric component, while for practical actuation, its output characteristics are also important.

To investigate the actual performances of the actuator, its working features were evaluated by the electric-fieldinduced displacement method. The test systems are composed of a drive module and a laser micromovement measurement module (Fig. 5a, see EXPERIMENTAL SECTION and Fig. S5 for details). The actuator is driven by two-channel signals with inverse phases and commonly electrical ground. Reference zero potential is provided to side terminal ports which are connected to all inner full electrodes, and other partitioned electrodes are
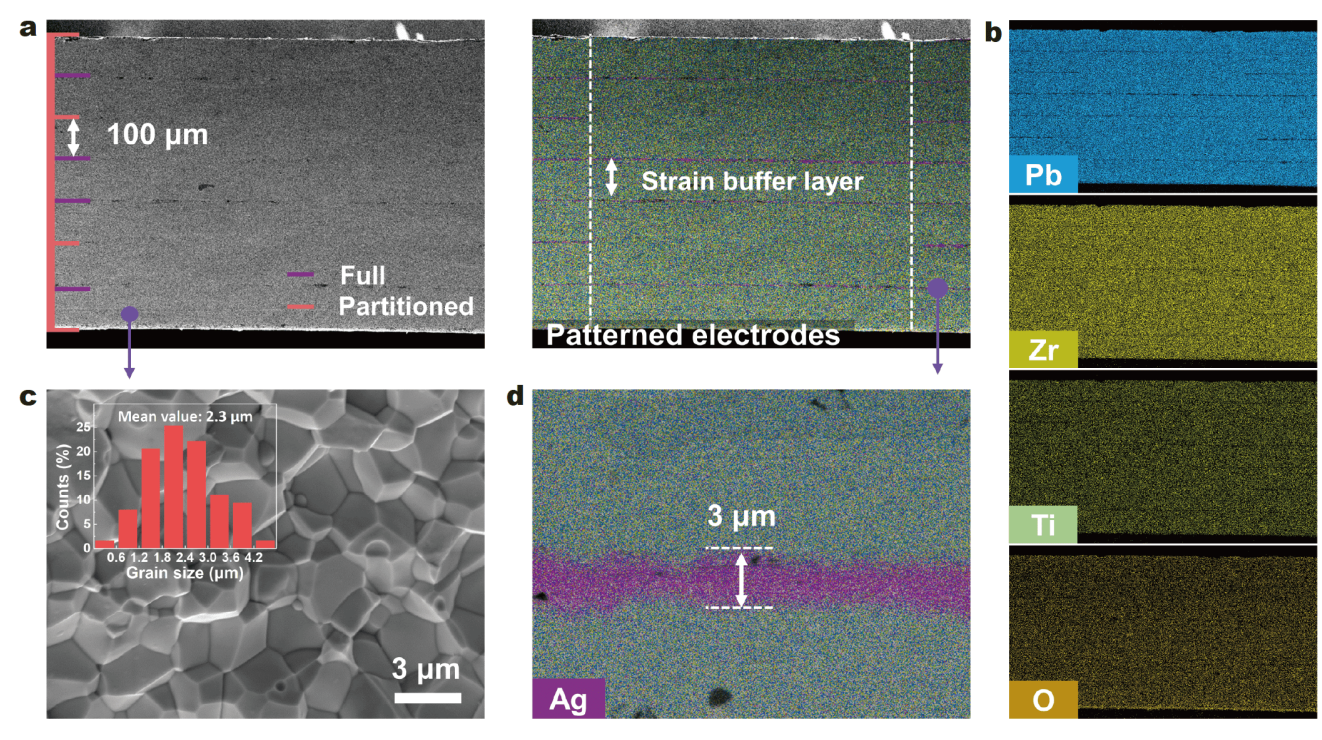

Figure 3 Microscopic structures and morphologies of the prototype cofired actuator specimen. (a, b) SEM and EDS results of a polished cross section demonstrate a monolithic structure composed of seven uniform layers each around $100 \mu \mathrm{m}$ thick, and two types of patterned electrodes are well embedded and aligned in order as design. (c, d) Amplified microregion denotes finely crystallized piezoceramics with an average grain size of about $2.3 \mu \mathrm{m}$ and well space-confined electrodes with a thickness of $3 \mu \mathrm{m}$. 


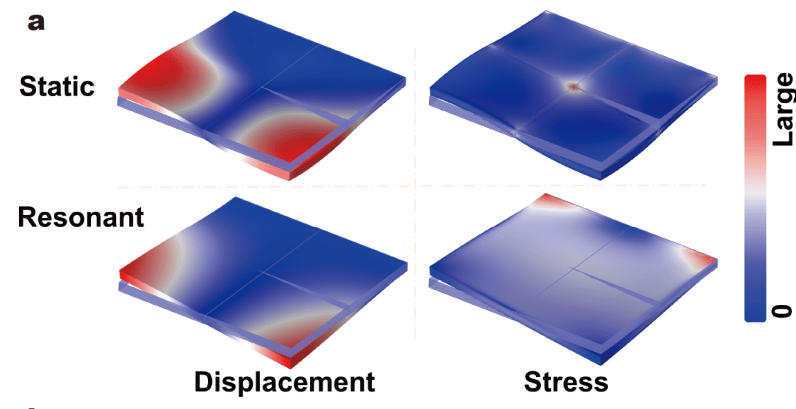

b
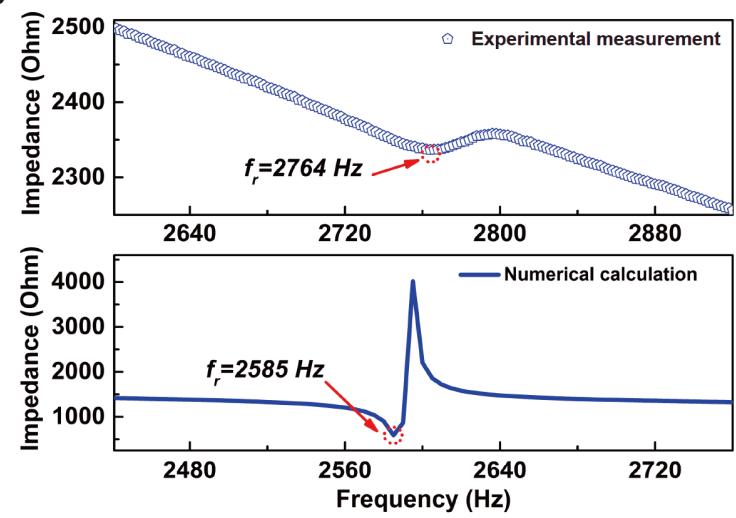

Figure 4 Twist deformation behaviors and impedance response on resonant states. (a) Finite element analyses show that the artificial twist modes are excited on both quasi-static and resonant states, and von Mises stress distribution is also displayed. (b) Impedance response of near-resonant state is calculated and measured, and the resonant frequencies match well.

driven as the way illustrated in Fig. 1e. One side surface is fixed and the transverse twist deflection of the edge point at another mechanically free side behaves as effective outputs. Fig. 5b measures the real-time twist displacement answers under various values of sinusoidal drive signals at $1 \mathrm{~Hz}$. The curves show the timely sinusoidal motion without obvious distortion or relaxation. An ideal sizable amplitude around $\pm 3.7 \mu \mathrm{m}$ is detected under peakto-peak values of $80 \mathrm{~V}$. It should be noted that a highly negative correlation between the displacement response and layer thickness exists (Fig. 2), and for industrialized LTCC technology, the thickness of each layer can be easily controlled to tens of microns or thinner, so if necessary, larger outputs (tens of micros) could be obtained under even milder drive voltages. The synergetic but anisotropic deformation of piezoceramic strain subunits may result in sharp stress change or concentration at interfaces, and dimmish device performance and service life. For practical applications, reasonable structures to prevent these potential threats should be elaborately designed, such as the stress release sites in classical multi- layer morphologies, or the strain buffer layer in this work.

Fig. $5 \mathrm{c}$ adds real-time actuation performance at $0.1 \mathrm{~Hz}$, and gets slightly bigger values (around $\pm 3.85 \mu \mathrm{m}$ under $\pm 40 \mathrm{~V})$. As an electromechanical elastomer, macroscopic strains of a piezoelectric actuator mainly arise from two major factors, which are the microscopic stretch response of dipole polarization and energy-driven domain wall evolutions. Lower frequencies provide the relaxation process with a more sufficient reaction time, so to some extent, displacement outputs reasonably increase. Fig. 5d, e plot actuation behaviors as a function of various zigzag drive signals at 1 and $0.1 \mathrm{~Hz}$, respectively. A mild asymmetric distortion is supposed to originate from the phase hysteresis relative to the drive voltages induced by the capacitance characteristics of piezoceramics. But overall, these data manifest basically a good linear relationship between the displacement output and drive signals, which guarantees controllability and practicability of twist actuation. Fig. $5 f$ summarizes the displacement amplitudes as a function of applied electric fields (or voltages). The measured answers are essentially proportional to the drive signal strength and also, match the calculated linear prediction very well. Strain behaviors come from linear piezoelectric parts and nonlinear parts of ferroelectric domain evolution and electrostriction effect (under fairly large electric fields), and the nonlinear parts cause reasonable deviations between simulated and experimental results. These results denote that not only has the novel design achieved twist deformation in a monolithic cofired piezoelectric component, but also its practical performance is qualified to offer sizable and reliable twist actuation.

\section{CONCLUSIONS}

In this study, we have developed a novel design to firstly realize fundamental twist mode from quasi-static to broadband frequencies in monolithic cofired piezoelectric components. Compared with classical twist actuators, this kind of device is completely compatible with industrialized LTCC technology, and meets integrated preparation and mass production requirements. Tough long-term technique difficulties such as output hysteresis, serving fatigue caused by structural adhesives promise to be initially solved. Twist output characteristics of the components are easy to be manipulated by optimizing geometrical sizes. A stable linear displacement output to applied electric fields is experimentally demonstrated. Moreover, the methodology here essentially opens up a new avenue for advancing future brand-new piezoceramic actuation technologies. By stacking identical ac- 
a

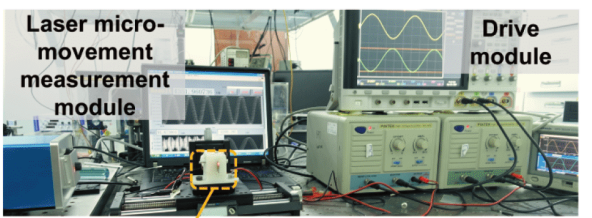

Optical platform

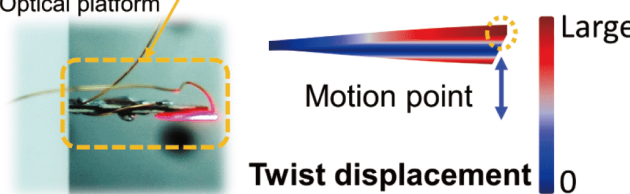

Twist displacement 0
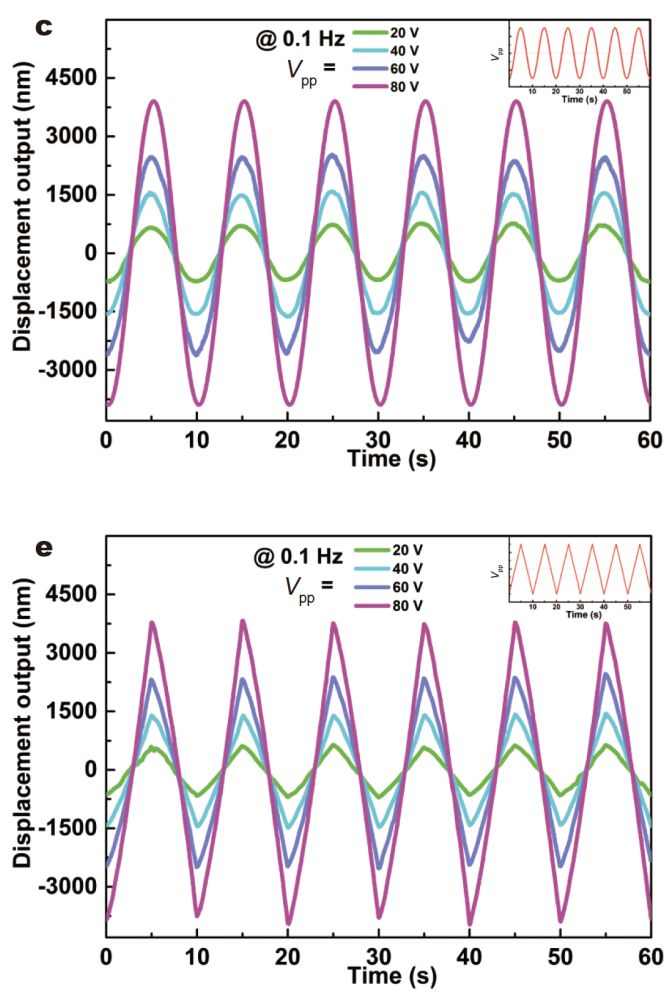
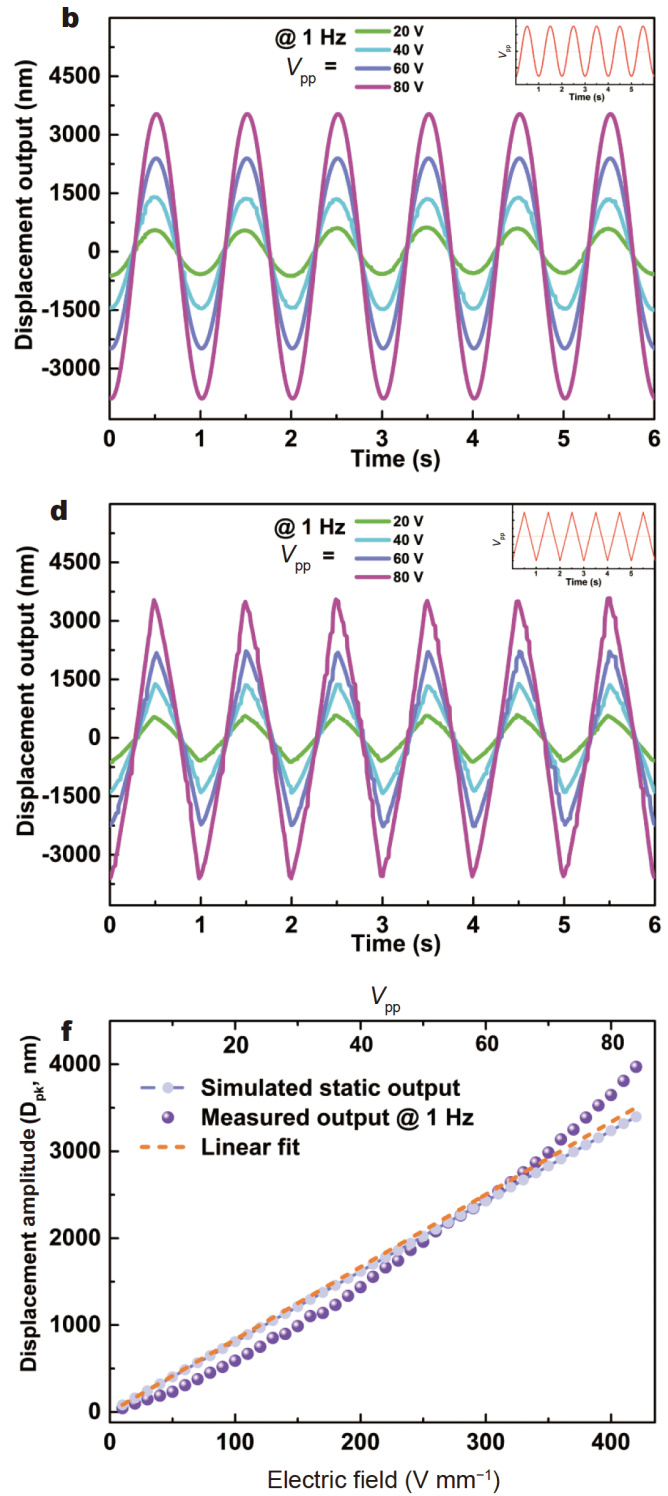

Figure 5 Deflection displacement outputs of the twist actuator are investigated by the electric-field induced displacement method. (a) The measurement system is composed of drive parts and testing parts, and a timely response of one edge point on the free end is measured. (b-d) Real-time displacement answers under sinusoidal and zigzag drive signals with various voltage values at 1 and $0.1 \mathrm{~Hz}$. (e) Dependence of the measured displacement amplitudes on the applied electric fields (or voltages). The results are basically linear and agree well with the simulated one.

tuation subunits in spatial order, tailoring patterned electrode morphologies, and integrating them in one component with the aid of LTCC techniques, actuation elements with objective arbitrary mode response at wide broadbands should become a reality.

Received 9 February 2021; accepted 24 March 2021; published online 16 June 2021

1 Shayegan M, Karrai K, Shkolnikov YP, et al. Low-temperature, in situ tunable, uniaxial stress measurements in semiconductors using a piezoelectric actuator. Appl Phys Lett, 2003, 83: 5235-5237

2 Masmanidis SC, Karabalin RB, De Vlaminck I, et al. Multifunctional nanomechanical systems via tunably coupled piezoelectric actuation. Science, 2007, 317: 780-783

3 Gao X, Yang J, Wu J, et al. Piezoelectric actuators and motors: Materials, designs, and applications. Adv Mater Technol, 2020, 5: 1900716

4 Liu Q, Zhang Y, Gao J, et al. High-performance lead-free piezoelectrics with local structural heterogeneity. Energy Environ Sci, 2018, 11: 3531-3539

5 Chen Z, Li F, Huang Q, et al. Giant tuning of ferroelectricity in single crystals by thickness engineering. Sci Adv, 2020, 6: eabc7156 
6 Qi H, Xie A, Tian A, et al. Superior energy-storage capacitors with simultaneously giant energy density and efficiency using nanodomain engineered $\mathrm{BiFeO}_{3}-\mathrm{BaTiO}_{3}-\mathrm{NaNbO}_{3}$ lead-free bulk ferroelectrics. Adv Energy Mater, 2020, 10: 1903338

7 Liu Y, Guo J, Yu A, et al. Magnetic-induced-piezopotential gated $\mathrm{MoS}_{2}$ field-effect transistor at room temperature. Adv Mater, 2018, 30: 1704524

8 Yang J, Huan Q, Yu Y, et al. Tailoring artificial mode to enable cofired integration of shear-type piezoelectric devices. Adv Sci, 2020, 7: 2001368

9 Benjeddou A. Shear-mode piezoceramic advanced materials and structures: A state of the art. Mech Adv Mater Struct, 2007, 14: 263-275

10 Liu J, Tian H, Lucas E, et al. Monolithic piezoelectric control of soliton microcombs. Nature, 2020, 583: 385-390

11 Cheng $\mathrm{T}, \mathrm{Li} \mathrm{H}, \mathrm{He} \mathrm{M}$, et al. Investigation on driving characteristics of a piezoelectric stick-slip actuator based on resonant/off-resonant hybrid excitation. Smart Mater Struct, 2017, 26: 035042

12 Liu J, Liu Y, Zhao L, et al. Design and experiments of a single-foot linear piezoelectric actuator operated in a stepping mode. IEEE Trans Ind Electron, 2018, 65: 8063-8071

13 Sung CC, Varadan VV, Bao XQ, et al. Active torsional vibration control experiments using shear-type piezoceramic sensors and actuators. J Intelligent Material Syst Struct, 1994, 5: 436-442

14 Zhu ML, Lee SWR, Li HL, et al. Modeling of torsional vibration induced by extension-twisting coupling of anisotropic composite laminates with piezoelectric actuators. Smart Mater Struct, 2002, 11: 55-62

15 Thakkar D, Ganguli R. Helicopter vibration reduction in forward flight with induced-shear based piezoceramic actuation. Smart Mater Struct, 2004, 13: 599-608

16 Hu G, Liang J, Lan C, et al. A twist piezoelectric beam for multidirectional energy harvesting. Smart Mater Struct, 2020, 29: 11LT01

17 Yang J, Chen Z, Hu Y. Trapped thickness-twist modes in an inhomogeneous piezoelectric plate. Philos Mag Lett, 2006, 86: 699705

18 Frenzel T, Kadic M, Wegener M. Three-dimensional mechanical metamaterials with a twist. Science, 2017, 358: 1072-1074

19 Shen Q, Trabia S, Stalbaum T, et al. A multiple-shape memory polymer-metal composite actuator capable of programmable control, creating complex 3D motion of bending, twisting, and oscillation. Sci Rep, 2016, 6: 24462

20 Frenzel T, Hahn V, Ziemke P, et al. Large characteristic lengths in 3D chiral elastic metamaterials. Commun Mater, 2021, 2: 4

21 Mohammadi F, Kholkin AL, Jadidian B, et al. High-displacement spiral piezoelectric actuators. Appl Phys Lett, 1999, 75: 2488-2490

22 Pan CL, Feng ZH, Ma YT, et al. Small torsional piezoelectric fiber actuators with helical electrodes. Appl Phys Lett, 2008, 92: 012923

23 Glazounov AE, Zhang QM, Kim C. Piezoelectric actuator generating torsional displacement from piezoelectric $d_{15}$ shear response. Appl Phys Lett, 1998, 72: 2526-2528

24 Berik P, Benjeddou A. Piezoelectric $\mathrm{d}_{15}$ shear response-based torsion actuation mechanism: An experimental benchmark and its 3D finite element simulation. Int J Smart Nano Mater, 2010, 1: 224235

25 Berik P, Chang WY, Jiang X. Piezoelectric $\mathrm{d}_{36}$ in-plane shear-mode of lead-free BZT-BCT single crystals for torsion actuation. Appl Phys Lett, 2017, 110: 052902

26 Trotta R, Atkinson P, Plumhof JD, et al. Nanomembrane quan- tum-light-emitting diodes integrated onto piezoelectric actuators. Adv Mater, 2012, 24: 2668-2672

27 Yan Y, Zhou Y, Priya S. Giant self-biased magnetoelectric coupling in co-fired textured layered composites. Appl Phys Lett, 2013, 102: 052907

28 Ma M, Wang Y, Navarro-Cía M, et al. The dielectric properties of some ceramic substrate materials at terahertz frequencies. J Eur Ceramic Soc, 2019, 39: 4424-4428

29 Li Z, Gao X, Yang J, et al. Designing ordered structure with piezoceramic actuation units (OSPAU) for generating continual nanostep motion. Adv Sci, 2020, 7: 2001155

30 Zhao P, Cai Z, Chen L, et al. Ultra-high energy storage performance in lead-free multilayer ceramic capacitors via a multiscale optimization strategy. Energy Environ Sci, 2020, 13: 4882-4890

31 Li J, Shen Z, Chen X, et al. Grain-orientation-engineered multilayer ceramic capacitors for energy storage applications. Nat Mater, 2020, 19: 999-1005

32 Yang J, Li Z, Xin X, et al. Designing electromechanical metamaterial with full nonzero piezoelectric coefficients. Sci Adv, 2019, 5 : eaax 1782

33 Chen K, Li L. Ordered structures with functional units as a paradigm of material design. Adv Mater, 2019, 31: 1901115

34 Pan E, Bai G, Ma B, et al. Reversible enhanced upconversion luminescence by thermal and electric fields in lanthanide ions doped ferroelectric nanocomposites. Sci China Mater, 2020, 63: 110-121

35 PourhosseiniAsl MJ, Gao X, Kamalisiahroudi S, et al. Versatile power and energy conversion of magnetoelectric composite materials with high efficiency via electromechanical resonance. Nano Energy, 2020, 70: 104506

Acknowledgements This work was supported by the National Natural Science Foundation of China $(51772005,51132001$, and 52032012) and Beijing Key Laboratory for Magnetoelectric Materials and Devices.

Author contributions Yang J conceived the idea, designed the project, and carried out the experiments. Zhang $\mathrm{F}$ and $\mathrm{Li} \mathrm{Y}$ assisted in device preparation and testing. Dong $\mathrm{S}$ and Liu $\mathrm{Z}$ provided support and guidance for this project. The manuscript was prepared and written by Yang J.

Conflict of interest The authors declare that they have no conflict of interest.

Supplementary information Supporting data are available in the online version of the paper.

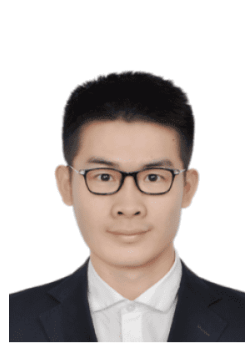

Jikun Yang is currently pursuing the $\mathrm{PhD}$ degree in advanced materials and mechanics at Peking University, Beijing, China. He received the BE degree in physics from the University of Science and Technology Beijing, China, in 2016. His research interests include piezoelectric materials and devices, metamaterials and $3 \mathrm{D}$ printing ceramics. 


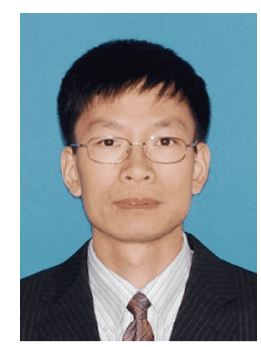

Zhifu Liu received his $\mathrm{PhD}$ degree from Shanghai Institute of Ceramics, Chinese Academy of Sciences (SICCAS) in 2004. He started his work at SICCAS from 2010. Now he is a group leader and deputy director of the Information Functional Materials and Devices Research Center, SICCAS. His research interests include microwave dielectric materials, lowtemperature cofired ceramics, multilayered ceramic components, and lead-free piezoelectric ceramics.

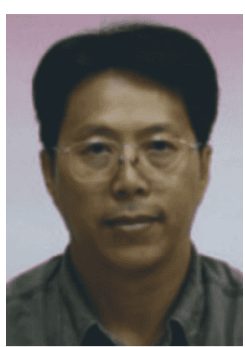

Shuxiang Dong is a tenured professor of materials science and engineering at the College of Engineering, Peking University, China. He received his master degree and doctor degree from Tsinghua University, China, in 1989 and 1993, respectively. Professor Dong's research focuses on piezoelectric ceramics and magnetoelectric composite materials, piezoelectric actuators and micromotors, magnetic sensors, smart electronic devices, and their applications.

\section{独石结构压电陶瓷扭转驱动器}

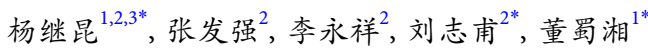

摘要 通过设计由异质单元组成的人工结构并调节其界面耦合方 式, 经常能够得到具有全新性质的功能器件. 对压电器件来说, 其变 形响应来自压电晶体的伸长、压缩、剪切模态及其组合, 同时取 决于结构设计的边界条件. 然而, 由于受到晶体微观层面镜面对称 性对扭转模态的限制, 长期以来, 在单一的集成压电元器件中实现 基本扭转驱动是一个很困难的任务. 本工作中, 我们设计了一种共 烧独石驱动器, 能够初次解决这个问题. 该原型器件由两组工作在 反向人工剪切模态的多层驱动单元组成, 它们手性的协同应力耦 合将在宽频范围内产生合成的扭转输出. 有限元模拟表明通过改 变其几何尺寸, 扭转位移可以在很大范围调节. 横向挠度测试证实 了其在电场作用下稳定且相当大的线性输出响应 $(1 \mathrm{~Hz}$ 和 $40 \mathrm{~V}$ 电压 条件下约 $3.7 \mu \mathrm{m}$ ). 更重要的是, 该设计方法实际上提出了在单一压 电陶瓷元件中获得任意模态和驱动状态的一种更广泛的途径. 